\title{
Novel Anti-Melanoma Immunotherapies: Disarming Tumor Escape Mechanisms
}

\author{
Sivan Sapoznik, ${ }^{1}$ Ohad Hammer, ${ }^{2}$ Rona Ortenberg, ${ }^{1,3}$ Michal J. Besser, ${ }^{1,3}$ \\ Tehila Ben-Moshe, ${ }^{4}$ Jacob Schachter, ${ }^{1}$ and Gal Markel ${ }^{1,3,5}$ \\ ${ }^{1}$ Ella Institute for Melanoma, Sheba Medical Center, Ramat Gan 52621, Israel \\ ${ }^{2}$ Pontifax Venture, Herzliya, Israel \\ ${ }^{3}$ Department of Clinical Microbiology and Immunology, Sackler Faculty of Medicine, Tel Aviv University, Ramat Aviv 61390, Israel \\ ${ }^{4}$ cCAM Biotherapeutics Ltd., Kiryat Shmona 11013, Israel \\ ${ }^{5}$ Talpiot Medical Leadership Program, Sheba Medical Center, Ramat-Gan 52621, Israel
}

Correspondence should be addressed to Gal Markel, markel@post.tau.ac.il

Received 24 January 2012; Accepted 8 April 2012

Academic Editor: Senthamil R. Selvan

Copyright (c) 2012 Sivan Sapoznik et al. This is an open access article distributed under the Creative Commons Attribution License, which permits unrestricted use, distribution, and reproduction in any medium, provided the original work is properly cited.

The immune system fights cancer and sometimes temporarily eliminates it or reaches an equilibrium stage of tumor growth. However, continuous immunological pressure also selects poorly immunogenic tumor variants that eventually escape the immune control system. Here, we focus on metastatic melanoma, a highly immunogenic tumor, and on anti-melanoma immunotherapies, which recently, especially following the FDA approval of Ipilimumab, gained interest from drug development companies. We describe new immunomodulatory approaches currently in the development pipeline, focus on the novel CEACAM1 immune checkpoint, and compare its potential to the extensively described targets, CTLA4 and PD1. This paper combines multidisciplinary approaches and describes anti-melanoma immunotherapies from molecular, medical, and business angles.

\section{Introduction}

The interplay between cancer cells and the host immune system displays an intriguing, dynamic battle for life. The current dogma on tumor progression under immune pressure is of the three "E"s: elimination, equilibrium, and escape [1]. In the first phase, the innate and adaptive immune system tracks and eliminates nascent tumor cells (immune surveillance). If not all cancer cells are eliminated, the second phase is equilibrium between cancer and the immune system, in which for a while, sometimes lasting years, the tumor remains dormant. This equilibrium, however, is temporary as genetic instability of cancerous cells together with continuous pressure of immune cells gradually shapes the immunogenicity of the tumor, transforming it into poorly immunogenic. This process, called immune editing, leads eventually to tumor escape and thereby progression into clinically evident disease. The immune system thus suppresses tumors on the one hand while promoting it on the other hand, by selecting and encouraging poorly-immunogenic variants (reviewed in
[1-3]). The mechanisms of tumor escape are numerous. They include alteration of the features of the tumor cells themselves (up-regulation of anti-apoptotic molecules and of cytotoxic determinants and downregulation of antigen presentation MHC molecules), secretion of cytokines that inhibit effective immune response (e.g., VEGF, IL-10, and $\operatorname{TGF} \beta$ ), and the induction of an immuno suppressive environment by indoleamine 2,3 dioxygenase (IDO) or via recruitment of inhibitory immune cells (Treg, MDSC, NKT, iDC, and macrophages) [4-6].

We will focus here on metastatic melanoma, which is an excellent example for the above mentioned model, as it is highly immunogenic and responds to immunotherapy [7]. Malignant melanoma is a main cancer-related cause of death in people below thirty. It is the most rapidly increasing malignancy in Western population in terms of incidence and is currently the sixth most common cancer in the USA, displaying high mortality rate, surpassed only by lung cancer $[8,9]$. As surgery is beneficial only for localized (primary) melanoma, continuous efforts are made to find effective 
immunotherapies for metastatic melanoma (MM). Systemic treatments include the administration of nonspecific immune-stimulating cytokines [7], immunization with cancer cells or molecules [10], adoptive $\mathrm{T}$ cell transfer [11], the recently developed small inhibitors of melanoma oncogenes [12], and blocking antibodies against inhibitory immune molecules [13]. Accumulating data proved that melanoma induces both innate and adaptive immune responses and that immune cells home to and infiltrate melanoma masses. However, the avidity of these cells is probably low, due to low cell number, low cytotoxic potential, or inhibitory microenvironment [14-17]. We will here describe promising immune treatments that aim to enhance the naturally occurring anti-melanoma immune response.

\section{Anti-Melanoma Immunotherapies}

2.1. Anti-CTLA4 (Ipilimumab and Tremelimumab). CTLA4 is an inhibitory molecule expressed on T cells undergoing activation, which functions to prevent prolonged activation signals. $\mathrm{T}$ cells are activated by two sequential signals: antigen recognition (TCR binding to antigen/MHC on APCs) and costimulation (e.g., CD28 interaction with B7.1 or B7.2 on APCs). CTLA-4 competes with CD28 on the binding of $\mathrm{B} 7$ and, when upregulated, inhibits CD28-dependent proliferation and activation and instead leads to cell cycle arrest, decreased cytokine production, and IDO secretion from APCs $[18,19]$. Noteworthy, it was reported that CTLA4 is also expressed by various tumor cells [20] and in a Wnt-dependent manner in melanoma [21]. Stimulation of tumor-expressed CTLA-4 with soluble ligands or agonistic mAb leads to induction of apoptosis $[20,21]$ as well as inhibition of proliferation and secretion of angiogenic cytokines [22]. These observations point out that CTLA4 exerts nonimmune-related functions when expressed by nonlymphoid cells. It could also reflect a yet undefined mechanism by which tumors achieve an "immune escape" phenotype and actively suppress, evade, and avoid $\mathrm{T}$ cell immunity [23].

Complete knockout of CTLA-4 is lethal, and mice suffer from massive lymphoproliferation and organ destruction $[24,25]$. However, preclinical studies showed that blocking of CTLA-4 results in anti-tumor activity and tumor regression in many mice tumor models (prostate, breast, lymphoma, melanoma) [26-29], which paved the way for clinical studies. Two anti-CTLA-4 monoclonal antibodies, generated by different companies, were tested in clinical trials in MM patients: Tremelimumab (Pfizer) and Ipilimumab/Yervoy (Bristol Myers Squibb), but only the latter was successful in phase III studies. Based on its ability to prolong survival of previously treated as well as untreated MM patients [30,31], Ipilimumab gained European Union (2010) [32] and FDA (March 2011) approval.

Two exciting phase III studies tested the clinical effects of Ipilimumab in advanced MM patients. In the first, 676 participants from 125 different medical centers that were already treated with standard treatments received either Ipilimumab, gp100 vaccine, or the combination of both, in a randomized, double-blind manner. Treatment with Ipilimumab improved median overall survival rates (10.0 and 10.1 months in the Ipilimumab-treated groups as compared with 6.4 months in the gp100-only treated group). The percentages of the patients who responded to Ipilimumab in the two groups were very limited (complete response in $\sim 1 \%$ and partial response in 5-10\%), but the effects of response were long-lasting in the majority of the responders [30]. In the second trial [31], 502 patients that were not previously treated received either dacarbazine (DTIC, standard care chemotherapy) or Ipilimumab in combination with dacarbazine in a double-blind, placebo-controlled manner. In this experiment, Ipilimumab increased overall survival rates from 9.1 to 11.2 months and 3-year survival from $12.2 \%$ to $20.8 \%$. Adverse effects, mainly immune related in the skin and gastro-intestinal track, accompanied nearly all patients in the two trials, with about half of the patients suffering from severe adverse effects in the second trial and several severe immune effects-related deaths in the first trial. These exciting results thus also exhibit the complicity of specifically manipulating immune responses.

2.2. Anti-PD1 (MDX-1106 and CT-1101). PD-1, as CTLA-4, is an inhibitory receptor belonging to the $\mathrm{CD} 28$ superfamily of immune-regulatory receptors. However, while CTLA-4 expression is limited to T cells, $\mathrm{PD}-1$ has a broader expression profile and is expressed on activated T, B and several myeloid cells. PD-1 (programmed death 1) downregulates T cell function (proliferation, cytokine secretion, and cytolysis of target cells) by delivering negative signals upon binding to its ligands, PD-L1 and PD-L2 (reviewed in [33]). PD-L2 expression is restricted to APCs (dendritic cells and monocytes) [34-36], and it is involved in tolerance of T cells to environmental (e.g., orally administrated) antigens [37]. PD-L1, on the contrary, is expressed by multiple normal and cancerous tissues and confers peripheral tolerance from "self" antigens $[38,39]$. Upon normal levels of antigen exposure, PD-1 functions as a "gate keeper" to attenuate immune responses (reviewed in [40]). The importance of PD-1 is manifested in PD-1-deficient mice, which suffer from auto-immunities $[41,42]$. Upon abnormal antigen exposure levels (chronic viral infection, caner) however, this immune tolerance becomes a stumbling block, as PD-1 delivers "veto" signals for CTLs, a response which renders tumor cells protected from cytotoxic immune cells and hampers anti-tumor immune interventions, such as vaccinations and ACT [40]. PD-L1 is upregulated in cancerous cells in vitro by immune cytokines, which are critical for $\mathrm{T}$ cell functioning, such as IFN $\gamma$ [43], which may even positively feedback to enhance immune tolerance in vivo. Indeed, PD1-deficient mice exhibit enhanced anti-tumor $\mathrm{T}$ cell responses towards solid and hematopoietic tumor, including melanoma, these mice survive longer and the tumors are regressed [39, 44, 45] and tumor transduced to overexpress PD-L1 grew more aggressively in vivo [46]. Blocking the PD1/PD-L1 pathway delays tumor progression [39, 44, 47-49] and adoptive transfer of tumor-specific PD-1-deficient $\mathrm{T}$ cell receptor transgenic $\mathrm{T}$ cells can reject tumors [43]. In melanoma patients, PD-L1 is expressed on melanoma cells and the levels of PD-L1 expression positively correlate with overall 
survival [50]. PD-1 is upregulated in CD- $8^{+} \mathrm{T}$ cells from melanoma patients during the metastatic (III, IV) stages of disease [50] and this upregulation may be associated with $\mathrm{T}$ cell dysfunction [51].

In order to block the inhibitory PD-1/PD-L1 pathway, two different anti-PD-1 monoclonal inhibitory antibodies were generated, MDX-1106 (BMS-936558) [52] and CT-011 [53]. Phase I clinical studies with each of the antibodies proved their safety, well-tolerated administration, and limited toxicity (though in both of them the maximum tolerated dose was not reached) and provided pharmacokinetic data $[52,53]$. In these clinical experiments, MDX-1106 (fully human antibody) was assayed in 39 patients with advanced melanoma, colorectal cancer, prostate cancer, non-small-cell lung cancer and renal cell carcinoma [52]. In the CT-011 study (humanized antibody), 17 patients were included, with leukemia, lymphoma, or multiple myeloma [53]. Clinical benefit was observed in both experiments $[52,53]$ and clinical responses correlated with the extent of PD-L1 expression on tumors [52]. Phase II clinical studies with MDX-1106 are ongoing with biweekly administration in metastatic nonsmall-cell lung cancer, renal cell carcinoma, prostate cancer and metastatic melanoma. They show limited toxicity, good tolerance (maximum tolerated dose (MTD) was not reached) and anti-tumor activity with $37.5 \%$ objective response in the total patients cohort (including 3 melanoma patients). One of the most impressive results was that all responses were highly durable and were still ongoing when publishing these preliminary results [54]. Phase II clinical trials with CT011 are also ongoing (http://www.clinicaltrials.gov/). Two other antibodies of the PD-1 pathway are under clinical development (currently recruiting participants for phase I studies): MK-3475 (anti-PD-1) and MDX-1105-01 (anti PD-L1) (http://www.clinicaltrials.gov/). The combination of anti-PD-1 and anti-CTLA-4 was tested in murine B16 melanoma model and found to be more effective in tumor regression as compared to each of the blocking antibodies alone [55]. A phase I clinical trial involving the two antibodies is ongoing, as well as a trial that combines MD-1106 with melanoma vaccines (http://www.clinicaltrials.gov/).

2.3. Comparison between Anti-CTLA-4 and Anti-PD-1. The different features of CTLA-4- as compared with PD-1deficient mice $[25,42]$ and the synergism of anti-CTLA4 and anti-PD-1 treatment in animal models [55] suggest that they act in distinct, non-redundant pathways. Though not enough experimental data using anti-PD-1 has been collected, the MTD of anti-PD-1 was not yet reached, and the drugs were not compared in a randomized manner, antiPD-1 seems to evoke less severe and less frequent adverse effects as compared with anti-CTLA-4 [52]. These differences may be attributed to the different cellular targets of the drugs. Anti-CTA-4 targets a peripheral interaction, between $\mathrm{T}$ cells and APCs. Thus, it is expected to cause general stimulation accompanied by adverse effects. The exact mechanism of action of MDX-1106 is not known. However, as it blocks the interactions of PD-1 with both PD-L1 and PD-L2 [52], it may act not only in the periphery but also within the tumor sites, interfering with $\mathrm{T}$ cell/tumor cell interactions and evoking specific, localized stimulation. In searching for localized immune modulators, which act within the tumor milieu and whose manipulation will not lead to severe autoimmunity, we have studied the roles of CEACAM1 in melanoma (Figure 1).

2.4. CEACAM1 as a Novel Immunotherapeutic Target. Carcinoembryonic antigen-related cell adhesion molecule 1 (CEACAM1, CD66a), a member of the Ig superfamily, is a broadly expressed, multifunctional, cell-cell adhesion molecule $[56,57]$. While not expressed in normal melanocytes [58], it is neoexpressed by the vast majority of melanoma specimens (unpublished observation) and is elevated during the histopathological progression of metastatic melanoma [59]. CEACAM1 is considered as an independent, highly significant marker for the development of melanoma metastases and poor survival [60]. Accumulating in vitro evidence suggests that it is not merely a marker but also confers cancerous characteristics to melanoma cells and thus may actively participate in the etiology of melanoma $[58,61]$. In the immune system, CEACAM1 acts as an inhibitory molecule that blocks proliferation and cytotoxic activity of T cells [62-64] and NK cells $[62,65-70]$ via ITIM sequences and the recruitment of SHP-1 and SHP-2 phosphatases [69, $71,72]$. Supporting this immune-inhibitory role, the expression of CEACAM1 on target cells, including melanoma, protects them from being eliminated in vitro by NK and T cells $[62,64,69]$. We have recently reported that melanoma cells that have survived an in-vitro $\mathrm{T}$ cell attack actively increase CEACAM1 expression in an IFN $\gamma$-dependent manner [64] and that this elevation enhances the protective effect against subsequent immune attacks [63]. Moreover, we could identify CEACAM1-positive NK cells in lymph nodes infiltrated with CEACAM1-positive melanoma cells, but not with CEACAM1-negative melanoma cells [69]. These data suggest a potentially novel tumor escape mechanism that could be used by CEACAM1-positive melanoma cells to evade elimination by transferring CEACAM1 to the attacking immune cells. Indeed, transfer of CEACAM1 was observed in-vitro, although it was considerably less efficient than transfer of CEACAM5 [73]. Importantly, patient-derived melanoma infiltrating lymphocytes [64] and circulating $\mathrm{T}$ and NK cells from melanoma patients [68] synthesize and express functional CEACAM1 $[64,68,69,74]$, which renders them susceptible to CEACAM1-mediated inhibition and may thus contribute to cancer progression. We have observed over-expression of CEACAM1 by circulating cytotoxic lymphocytes in other diseases, including ankylosing spondilitis and bare lymphocyte syndrome type I $[65,67,68]$, as well as on decidual lymphocytes obtained from CMV-infected pregnancies [62], all occurring due to yet to be defined mechanisms. These may be related to aberrant immune stimulation or to abnormal development of immune cells [68].

Based on these findings, we have developed a highaffinity murine monoclonal antibody against human CEACAM1 [75]. Anti-CEACAM1 does not act on CEACACM1positive cells in cis (i.e., does not interfere with general cellular processes such as proliferation and apoptosis). 


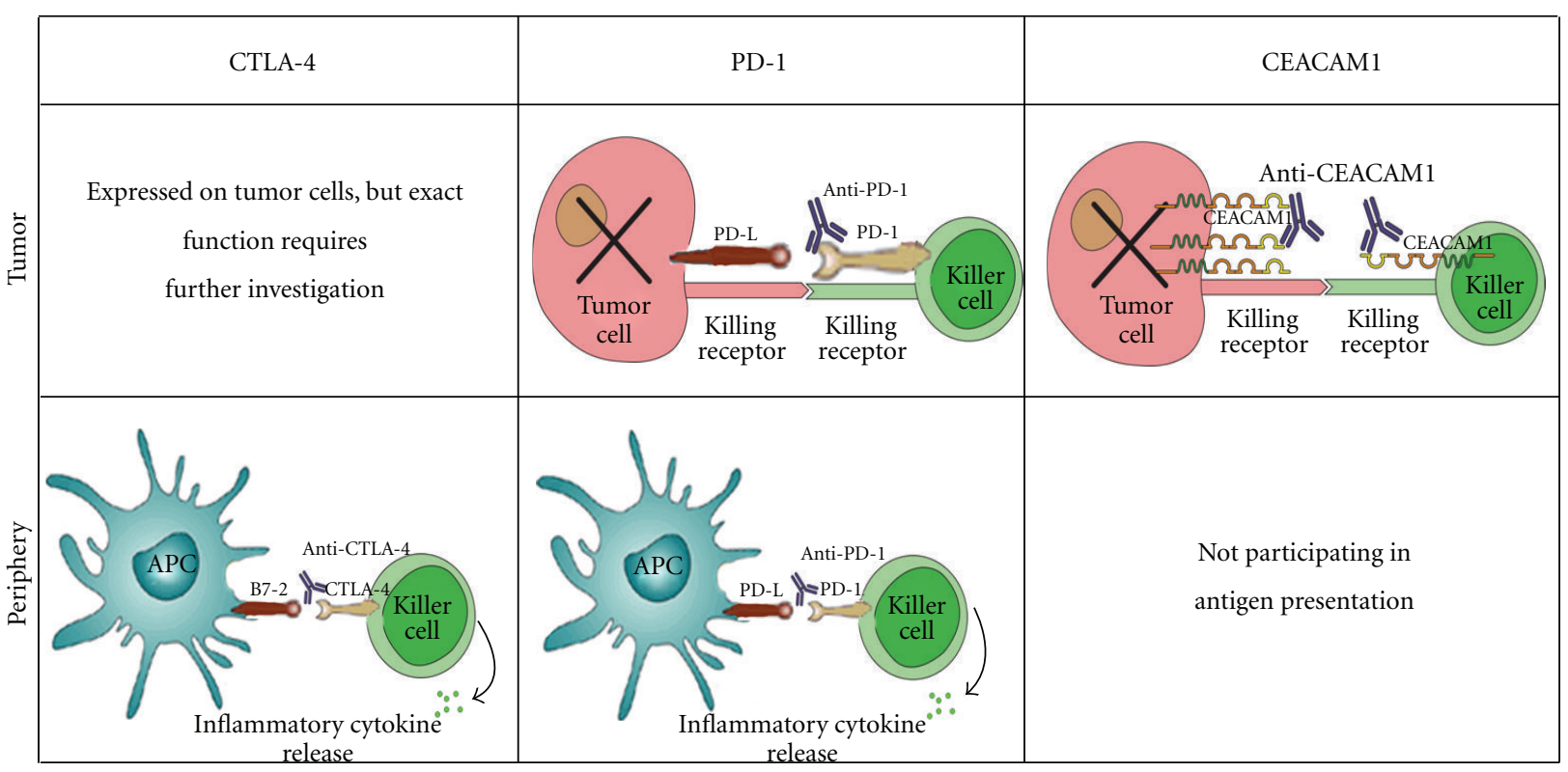

FIgURE 1: Sites of action for selected immune modulators. Anti-CTLA-4 targets the interactions between T cells and APCs in the periphery and thus is prone to evoke general stimulation accompanied by frequent adverse effects. Anti-PD-1 may block peripheral interactions, as anti-CTLA-4, but also the interactions between tumors and infiltrating immune killer cells, and thus may evoke both general and localized stimulation. CEACAM1 is expressed both on immune killer cells and on the tumor target, and anti-CEACAM1 is expected to act specifically at the interface between these two and to evoke low autoimmunity.

Rather, it acts in trans, binding both T cells and melanoma, to efficiently relieve the CEACAM1-dependent inhibition of $\mathrm{T}$ cell cytotoxicity. Therefore, the mechanism of action in vivo of anti-CEACAM1 strongly depends on the endogenous immune system and its ability to recognize the target cells in an antigen-restricted manner, thereby reducing the risk of adverse effects stemming from generalized non-specific immune stimulation. We showed that anti-CEACAM1 renders melanoma cells susceptible to elimination by $\mathrm{T}$ cells, both in-vitro and in a human-melanoma xenograft murine model, which maintains antigen-restricted recognition [75]. Indeed, we have previously shown that abolishment of CEACAM1 with polyclonal anti-CEACAM antibodies does not induce a nonspecific $\mathrm{T}$ cell function [64].

Several lines of evidence pointed to the potential high specificity of anti-CEACAM1 to the cancerous state and to its potentially low risk of evoking adverse effects: (a) staining of normal tissue micro-array with anti-CEACAM1 proved only limited staining of luminal cells of some secretory ducts. These patterns are substantially more restricted than staining patterns of other FDA-approved therapeutic antibodies, such as Erbitux; (b) the anti-CEACAM1 mAb does not elicit complement-dependent cytotoxic effect nor non-specific $\mathrm{T}$ cell activation; (c) the anti-CEACAM1 mAb is not an agonistic antibody and is therefore probably incapable of exerting direct functional effects on CEACAM1-positive cells. Rather, it is an antagonistic antibody, whose effects depend on antigenic recognition between $\mathrm{T}$ cells and their targets; (d) the immune-inhibitory homophilic CEACAM1 interactions are expected to take place only in the tumor, and not during earlier stages of the elicited immune response, such as antigen presentation. CEACAM1 homophilic interactions occur between CEACAM1-positive cancer cells and CEACAM1-positive tumor infiltrating lymphocytes, which are late-effector lymphocytes. Thus, blocking of CEACAM1 is expected to enhance the immune response only within tumor sites and only in the context of antigen-restricted recognition. These exciting results mark anti-CEACAM1 as a potential specific and safe (compartmentalized to the tumor vicinity) novel immunotherapeutic modality (Figure 1). Another important advantage of CEACAM1directed therapy is that patient selection would be based on the presence of CEACAM1 on tumor tissue. It should be noted that CEACAM1 is expressed in $60-80 \%$ of metastatic melanoma cases, which suggests that the majority of metastatic melanoma patients would benefit from anti-CEACAM1 antibodies. The anti-CEACAM1 approach is developed by cCAM BioTherapeutics, and first-in-man clinical trials are anticipated in the near future.

2.5. Adoptive T Cell Transfer (ACT). Adoptive cell therapy with ex vivo cultured T cells, developed by Rosenberg and his colleagues in the National Cancer Institute, is currently the most promising immunotherapy for MM patients, yielding $50-70 \%$ objective response rates $[76,77]$. It is based on the isolation of bulk $\mathrm{T}$ cell masses from resected melanoma, their ex-vivo expansion by about 1000-fold (reaching about $50 \times 10^{9}$ cells), and their reinfusion to the patient following lymphodepleting nonmyeloablative chemotherapy, which eliminates endogenous competitor immune cells [76, 77]. 
TABLE 1: Current clinical trials in melanoma using monoclonal antibodies.

\begin{tabular}{lcccc}
\hline Company & Antibody & Target & Function & Status \\
\hline & Antagonistic Abs. & & & \\
Bristol-Myers Squibb & Ipilimumab (Yervoy) & CTLA-4 & Relieve immune block & Approved \\
Bristol-Myers Squibb & MDX-1106 ${ }^{1}$ & PD-1 & Relieve immune block & Phase II (completed) \\
Curetech Ltd. (Israel) & CT-011 & PD-1 & Relieve immune block & Phase II (recruiting) \\
Merck & MK-3475 & PD-1 & Relieve immune block & Phase I (recruiting) \\
Bristol-Myers Squibb & MDX-1105-01 & PD-L1 & Relieve immune block & Phase I (recruiting) \\
& Agonistic Abs. & & & Phase II (completed) \\
Bristol-Myers Squibb & BMS-663513 & $4-1$ BB & Stimulate T cells & Phase I (recruiting) \\
Pfizer & CP870,893 & CD40 & Stimulate T cells & Phase I (on hold) \\
Tolerx & TRX518 & GITR & Inhibit T regs & Phase II (not open yet) \\
Portland Providence Medical Center & Anti-OX40 & OX40 & Stimulate T cells &
\end{tabular}

Recently, we have shown that $\mathrm{T}$ cells derived from enzymatic digestion of resected tumors (rather from multiple small fragments) yield high numbers in culture, which enable to shorten their ex-vivo culturing period [78]. We and others have shown that responding patients were treated with TIL that spent less time in culture $[78,79]$. Indeed, Young TIL cultures were successfully established for nearly $90 \%$ of MM patients, and overall response rates reached 50\% [78]. The main disadvantages of ACT are that the generation of TIL cultures presents a technical challenge and is labor, cost and time consuming $[76,77]$. Attempts to overcome several of these limitations by the usage of genetically-engineered rather than endogenous $\mathrm{T}$ cells were presented in two clinical trials. These trials, in which T cells were modified to overexpress TCR directed against melanoma antigen (MART-1 or gp100), yielded modest response rates (12-30\%) but proved the feasibility of the method $[80,81]$. Engineering T cells with chimeric antigen receptors (CARs), which recognize tumor cells in a MHC-independent manner and endow increased $\mathrm{T}$ cell activity [82], have been tested in preclinical studies in melanoma [83]. Recently, Peng and his colleagues have shown that over-expression of the murine chemokine receptor CXCR2 on T cells improves their homing to melanoma and tumor regression in mice model [84], suggesting that endowing $\mathrm{T}$ cells with improved chemotaxis capabilities to tumor sites may also enhance ACT.

2.6. Additional Antibodies. Additional immune-modulatory molecules that have gained scientific attention and are now under clinical development are OX40 (CD134), CD40, GITR, and 4-1BB (CD137) (http://www.clinicaltrials.gov/) (Table 1, also reviewed in [85]).

\section{Cancer Immunotherapy: Business Angle}

For decades, cancer immunotherapy has been neglected by drug development companies, which were deterred by the field unfavorable track record. Moreover, in order to achieve clinical proof of concept, immunotherapy requires a unique development strategy that involves long-term followup and randomized controlled studies. This stems in part from the fact that tumor shrinkage is less common with immunotherapy drugs coupled with the long period required for mounting a systemic immune response. [86]. Lastly, combining immune-modulating drugs with chemotherapy regimens was perceived as counterproductive.

This sentiment has gradually been changing following clinical validation with cancer vaccines and immunomodulatory antibodies. Of particular importance were FDA approvals for Sipuleucel-T and Ipilimumab, based on survival benefit in prostate cancer and melanoma, respectively, $[31,87]$. These agents demonstrated unequivocally the value of immunotherapy for cancer in broad unselected populations.

The renewed interest in cancer immunotherapy is best exemplified by recent deals involving clinical and preclinical programs. The growing number of transactions coupled with their lucrative financial terms serves as a testament to the excitement within the industry regarding harnessing the immune system to fight cancer. Although melanoma remains a common indication for immunotherapies, recent data clearly suggests that potential utility for this approach spans well beyond this indication.

\section{Deals}

In August 2010, Amplimmune licensed MP-224, an Fc-fused PD-L2, to GlaxoSmithKline (GSK). Amplimmune received an upfront payment of $\$ 23 \mathrm{M}$ and is eligible to receive $\$ 485 \mathrm{M}$ in milestone payments. MP-224 binds and inhibits the immunosuppressive activity of PD-1, a coinhibitory checkpoint on T cells [88]. The fusion protein is expected to be the fourth PD-1 neutralizing agent in clinical testing behind BMS' BMS-936558, Curetech's CT-011, and Merck's MK-3475.

In January 2011, Amgen acquired BioVex, which was developing OncoVex GM-CSF, a genetically modified herpes simplex virus 1 (HSV-1). The deal included \$425 M upfront 
and $\$ 575 \mathrm{M}$ of milestone payments. OncoVex GM-CSF is an oncolytic virus currently in phase III for the treatment of metastatic melanoma. The virus anti-cancer effect involves direct killing of tumor cells followed by immune activation that results from the virus immunogenicity and secretion of GM-CSF to the tumor microenvironment. In its phase II trial, OncoVex GM-CSF exhibited a unique clinical activity profile. Intratumor injection of the virus resulted in tumor shrinkage of injected as well as noninjected lesions. Responses were durable in a substantial portion of patients and overall survival was encouraging. An ongoing phase III trial is expected to generate results in 2012, using a primary endpoint of objective response lasting 6 months or more. Another phase III trial in head and neck cancer has been terminated in 2011.

In July 2011, BMS licensed IPH2102, an antibody targeting KIR receptors, from Innate Pharma. By binding the inhibitory KIR receptors on NK cells, the antibody, currently in phase I, is expected to promote an innate immune response against cancer cells. The deal included an upfront payment of $\$ 35 \mathrm{M}$ as well as $\$ 430 \mathrm{M}$ in development and commercialization milestones.

In September 2011, Bristol-Myers Squibb acquired exUS commercialization rights (except in Japan, Korea, and Taiwan) for BMS-936558 from Japan-based Ono Pharmaceuticals. BMS-936558 is a fully human antibody targeting PD-1, for which BMS had originally held US rights. In return, Ono received certain commercialization rights for abatacept (Fc-fused CTLA4) in Japan. BMS-936558 is the most advanced PD-1 inhibitor in clinical testing, currently studied in melanoma, lung, and renal cancer. Initial results with this antibody as a single agent are encouraging [89].

In October 2011, MedImmune (the biologics arm of AstraZeneca) in-licensed two programs in the field of cancer immunotherapy. One deal involved licensing tremelimumab, an anti-CTLA4 antibody from Pfizer. MeDimmune assumed global development rights for Tremelimumab, which failed a phase III trial in melanoma in 2008. Future development will likely be based on pharmacodynamic biomarkers identified retrospectively in the failed phase III study. Pfizer retained the rights to use drug with specified types of combination therapies. Terms of the agreement were not disclosed. A second deal was signed with Portland-based AgonOx, which is developing OX40 agonists for the treatment of cancer. AgonOx is developing Fc-fused OX40 ligand as well as agonist antibodies. A murine antibody against OX40 led to immune activation and tumor shrinkage in a phase I trial (company's web site).

In October 2011, Genesis Biopharma announced a deal with the NIH for patents covering TIL therapy. The deal included an upfront payment of $\$ 1.2 \mathrm{M}$ as well as undisclosed milestone payments and royalties. Following the deal, Genesis intends to turn the autologous cell-based treatment, which until now has been given as a service in medical centers, into a commercially available product. The company will offer the treatment, rebranded as Contigo, via several medical centers in the US and plans to manufacture it at a central production facility. The anticipated cost per patient is $\$ 120$ thousand, similar to that of ipilimumab.

\section{Disclosure}

G. Markel is the scientific founder of cCAM Biotherapeutics Ltd.

\section{Acknowledgments}

The authors thank the Lemelbaum family and Mr. Aaronson for their generous support. G. Markel is funded by Israel Cancer Association Grant 20112011, Israel Cancer Research Fund Grant 2011-719, Israel Science Foundation Grant 1489/10, and Israel Ministry of Infrastructure and Trade Grant 46201. The authors thank Dr. Ilana Mandel for her assistance.

\section{References}

[1] M. D. Vesely, M. H. Kershaw, R. D. Schreiber, and M. J. Smyth, "Natural innate and adaptive immunity to cancer," Annual Review of Immunology, vol. 29, pp. 235-271, 2011.

[2] S. A. Quezada, K. S. Peggs, T. R. Simpson, and J. P. Allison, "Shifting the equilibrium in cancer immunoediting: from tumor tolerance to eradication," Immunological Reviews, vol. 241, no. 1, pp. 104-118, 2011.

[3] R. D. Schreiber, L. J. Old, and M. J. Smyth, "Cancer immunoediting: integrating immunity's roles in cancer suppression and promotion," Science, vol. 331, no. 6024, pp. 1565-1570, 2011.

[4] T. J. Stewart and S. I. Abrams, "How tumours escape mass destruction," Oncogene, vol. 27, no. 45, pp. 5894-5903, 2008.

[5] I. Poschke, D. Mougiakakos, and R. Kiessling, "Camouflage and sabotage: tumor escape from the immune system," Cancer Immunology, Immunotherapy, vol. 60, no. 8, pp. 1161-1171, 2011.

[6] T. Yaguchi, H. Sumimoto, C. Kudo-Saito et al., "The mechanisms of cancer immunoescape and development of overcoming strategies," International Journal of Hematology, vol. 93, no. 3, pp. 294-300, 2011.

[7] C. M. Balch, A. N. Houghton, A. J. Sober, and S.-J. Soong, Eds., Cutaneous Melanoma, Quality Medical Publishing, 4th edition, 2003.

[8] A. Slominski, J. Wortsman, A. J. Carlson, L. Y. Matsuoka, C. M. Balch, and M. C. Mihm, "Malignant melanoma," Archives of Pathology and Laboratory Medicine, vol. 125, no. 10, pp. 12951306, 2001.

[9] A. Jemal, R. Siegel, E. Ward, T. Murray, J. Xu, and M. J. Thun, "Cancer statistics, 2007," Ca-A Cancer Journal for Clinicians, vol. 57, no. 1, pp. 43-66, 2007.

[10] O. Klein, C. Schmidt, A. Knights, I. D. Davis, W. Chen, and J. Cebon, "Melanoma vaccines: developments over the past 10 years," Expert Review of Vaccines, vol. 10, no. 6, pp. 853-873, 2011.

[11] S. A. Rosenberg, N. P. Restifo, J. C. Yang, R. A. Morgan, and M. E. Dudley, "Adoptive cell transfer: a clinical path to effective cancer immunotherapy," Nature Reviews Cancer, vol. 8, no. 4, pp. 299-308, 2008.

[12] C. Shepherd, I. Puzanov, and J. A. Sosman, "B-RAF inhibitors: an evolving role in the therapy of malignant melanoma," Current Oncology Reports, vol. 12, no. 3, pp. 146-152, 2010.

[13] J. Weber, "Immune checkpoint proteins: a new therapeutic paradigm for cancerpreclinical background: CTLA-4 and PD1 blockade," Seminars in Oncology, vol. 37, no. 5, pp. 430-439, 2010 . 
[14] J. Yuan, D. B. Page, G. Y. Ku et al., "Correlation of clinical and immunological data in a metastatic melanoma patient with heterogeneous tumor responses to ipilimumab therapy," Cancer Immunity, vol. 10, article 1, 2010.

[15] S. A. Rosenberg, R. M. Sherry, K. E. Morton et al., "Tumor progression can occur despite the induction of very high levels of self/tumor antigen-specific CD8 ${ }^{+} \mathrm{T}$ cells in patients with melanoma," Journal of Immunology, vol. 175, no. 9, pp. 61696176, 2005.

[16] A. Anichini, C. Vegetti, and R. Mortarini, "The paradox of T cell-mediated antitumor immunity in spite of poor clinical outcome in human melanoma," Cancer Immunology, Immunotherapy, vol. 53, no. 10, pp. 855-864, 2004.

[17] H. Harlin, T. V. Kuna, A. C. Peterson, Y. Meng, and T. F. Gajewski, "Tumor progression despite massive influx of activated $\mathrm{CD}^{+} \mathrm{T}$ cells in a patient with malignant melanoma ascites," Cancer Immunology, Immunotherapy, vol. 55, no. 10, pp. 1185-1197, 2006.

[18] M. L. Alegre, H. Shiels, C. B. Thompson, and T. F. Gajewski, "Expression and function of CTLA-4 in Th1 and Th2 cells," Journal of Immunology, vol. 161, no. 7, pp. 3347-3356, 1998.

[19] D. H. Munn, M. D. Sharma, and A. L. Mellor, "Ligation of B7-1/B7-2 by human CD4 ${ }^{+} \mathrm{T}$ cells triggers indoleamine 2,3dioxygenase activity in dendritic cells," Journal of Immunology, vol. 172, no. 7, pp. 4100-4110, 2004.

[20] E. Contardi, G. L. Palmisano, P. L. Tazzari et al., "CTLA4 is constitutively expressed on tumor cells and can trigger apoptosis upon ligand interaction," International Journal of Cancer, vol. 117, no. 4, pp. 538-550, 2005.

[21] K. V. Shah, A. J. Chien, C. Yee, and R. T. Moon, "CTLA-4 is a direct target of $\mathrm{Wnt} / \beta$-catenin signaling and is expressed in human melanoma tumors," Journal of Investigative Dermatology, vol. 128, no. 12, pp. 2870-2879, 2008.

[22] P. Queirolo, M. Boitano, P. Carrega et al., "Targeting CTLA-4 directly on melanoma cells: a possible novel perspective in the immunotherapy of cutaneous melanoma," Journal of Clinical Oncology, vol. 27, supplement, abstract e22138, 2009.

[23] J. S. Weber, "Tumor evasion may occur via expression of regulatory molecules: a case for CTLA-4 in melanoma," Journal of Investigative Dermatology, vol. 128, no. 12, pp. 2750-2752, 2008.

[24] E. A. Tivol, F. Borriello, A. N. Schweitzer, W. P. Lynch, J. A. Bluestone, and A. H. Sharpe, "Loss of CTLA-4 leads to massive lymphoproliferation and fatal multiorgan tissue destruction, revealing a critical negative regulatory role of CTLA-4," Immunity, vol. 3, no. 5, pp. 541-547, 1995.

[25] P. Waterhouse, J. M. Penninger, E. Timms et al., "Lymphoproliferative disorders with early lethality in mice deficient in Ctla-4," Science, vol. 270, no. 5238, pp. 985-988, 1995.

[26] J. A. van Ginderachter, Y. Liu, A. B. Geldhof, L. Brijs, K. Thielemans et al., "B7-1, IFN gamma and anti-CTLA-4 cooperate to prevent T-cell tolerization during immunotherapy against a murine T-lymphoma," International Journal of Cancer, vol. 87, pp. 539-547, 2000.

[27] A. A. Hurwitz, B. A. Foster, E. D. Kwon et al., "Combination immunotherapy of primary prostate cancer in a transgenic mouse model using CTLA-4 blockade," Cancer Research, vol. 60, no. 9, pp. 2444-2448, 2000.

[28] E. D. Kwon, A. A. Hurwitz, B. A. Foster et al., "Manipulation of $\mathrm{T}$ cell costimulatory and inhibitory signals for immunotherapy of prostate cancer," Proceedings of the National Academy of Sciences of the United States of America, vol. 94, no. 15, pp. 8099-8103, 1997.
[29] D. R. Leach, M. F. Krummel, and J. P. Allison, "Enhancement of antitumor immunity by CTLA-4 blockade," Science, vol. 271, no. 5256, pp. 1734-1736, 1996.

[30] F. S. Hodi, S. J. O’Day, D. F. McDermott et al., "Improved survival with ipilimumab in patients with metastatic melanoma," The New England Journal of Medicine, vol. 363, no. 8, pp. 711723, 2010.

[31] C. Robert, L. Thomas, I. Bondarenko et al., "Ipilimumab plus dacarbazine for previously untreated metastatic melanoma," The New England Journal of Medicine, vol. 364, no. 26, pp. 2517-2526, 2011.

[32] “Ipilimumab," Drugs in R \& D, vol. 10, no. 2, pp. 97-110, 2010.

[33] B. T. Fife and K. E. Pauken, "The role of the PD-1 pathway in autoimmunity and peripheral tolerance," Annals of the New York Academy of Sciences, vol. 1217, no. 1, pp. 45-59, 2011.

[34] M. Ishida, Y. Iwai, Y. Tanaka et al., "Differential expression of PD-L1 and PD-L2, ligands for an inhibitory receptor PD-1, in the cells of lymphohematopoietic tissues," Immunology Letters, vol. 84, no. 1, pp. 57-62, 2002.

[35] P. Loke and J. P. Allison, "PD-L1 and PD-L2 are differentially regulated by Th1 and Th2 cells," Proceedings of the National Academy of Sciences of the United States of America, vol. 100, no. 9, pp. 5336-5341, 2003.

[36] Y. Latchman, C. R. Wood, T. Chernova et al., "PD-L2 is a second ligand for PD-1 and inhibits T cell activation," Nature Immunology, vol. 2, no. 3, pp. 261-268, 2001.

[37] Y. Zhang, Y. Chung, C. Bishop et al., "Regulation of T cell activation and tolerance by PDL2," Proceedings of the National Academy of Sciences of the United States of America, vol. 103, no. 31, pp. 11695-11700, 2006.

[38] H. Dong, S. E. Strome, D. R. Salomao et al., "Tumor-associated B7-H1 promotes T-cell apoptosis: a potential mechanism of immune evasion," Nature Medicine, vol. 8, no. 8, pp. 793-800, 2002.

[39] Y. Iwai, M. Ishida, Y. Tanaka, T. Okazaki, T. Honjo, and N. Minato, "Involvement of PD-L1 on tumor cells in the escape from host immune system and tumor immunotherapy by PDL1 blockade," Proceedings of the National Academy of Sciences of the United States of America, vol. 99, no. 19, pp. 12293-12297, 2002.

[40] J. L. Riley, "PD-1 signaling in primary T cells," Immunological Reviews, vol. 229, no. 1, pp. 114-125, 2009.

[41] H. Nishimura, M. Nose, H. Hiai, N. Minato, and T. Honjo, "Development of lupus-like autoimmune diseases by disruption of the PD-1 gene encoding an ITIM motif-carrying immunoreceptor," Immunity, vol. 11, no. 2, pp. 141-151, 1999.

[42] H. Nishimura, T. Okazaki, Y. Tanaka et al., "Autoimmune dilated cardiomyopathy in PD-1 receptor-deficient mice," Science, vol. 291, no. 5502, pp. 319-322, 2001.

[43] C. Blank, I. Brown, A. C. Peterson et al., "PD-L1/B7H1 inhibits the effector phase of tumor rejection by $\mathrm{T}$ Cell receptor (TCR) transgenic CD8 ${ }^{+}$T cells," Cancer Research, vol. 64, no. 3, pp. 1140-1145, 2004.

[44] L. Zhang, T. F. Gajewski, and J. Kline, "PD-1/PD-L1 interactions inhibit antitumor immune responses in a murine acute myeloid leukemia model," Blood, vol. 114, no. 8, pp. 15451552, 2009.

[45] Y. Iwai, S. Terawaki, and T. Honjo, "PD-1 blockade inhibits hematogenous spread of poorly immunogenic tumor cells by enhanced recruitment of effector T cells," International Immunology, vol. 17, no. 2, pp. 133-144, 2005.

[46] Y. Iwai, S. Terawaki, M. Ikegawa, T. Okazaki, and T. Honjo, "PD-1 inhibits antiviral immunity at the effector phase in 
the liver," Journal of Experimental Medicine, vol. 198, no. 1, pp. 39-50, 2003.

[47] F. Hirano, K. Kaneko, H. Tamura et al., "Blockade of B7$\mathrm{H} 1$ and PD-1 by monoclonal antibodies potentiates cancer therapeutic immunity," Cancer Research, vol. 65, no. 3, pp. 1089-1096, 2005.

[48] S. E. Strome, H. Dong, H. Tamura et al., "B7-H1 blockade augments adoptive T-cell immunotherapy for squamous cell carcinoma," Cancer Research, vol. 63, no. 19, pp. 6501-6505, 2003.

[49] K. Durgan, M. Ali, P. Warner, and Y. E. Latchman, "Targeting NKT cells and PD-L1 pathway results in augmented antitumor responses in a melanoma model," Cancer Immunology, Immunotherapy, vol. 60, no. 4, pp. 547-558, 2011.

[50] H. Kronig, K. J. Falchner, M. Odendahl, B. Brackertz, H. Conrad et al., "PD-1 expression on melanA-reactive T-cells increases during progression to metastatic disease," International Journal of Cancer, vol. 130, no. 10, pp. 2327-2336, 2011.

[51] J. Fourcade, Z. Sun, M. Benallaoua et al., "Upregulation of Tim-3 and PD-1 expression is associated with tumor antigenspecific $\mathrm{CD}^{+} \mathrm{T}$ cell dysfunction in melanoma patients," Journal of Experimental Medicine, vol. 207, no. 10, pp. 21752186, 2010.

[52] J. R. Brahmer, C. G. Drake, I. Wollner et al., "Phase I study of single-agent anti-programmed death-1 (MDX-1106) in refractory solid tumors: safety, clinical activity, pharmacodynamics, and immunologic correlates," Journal of Clinical Oncology, vol. 28, no. 19, pp. 3167-3175, 2010.

[53] R. Berger, R. Rotem-Yehudar, G. Slama et al., "Phase i safety and pharmacokinetic study of CT-011, a humanized antibody interacting with PD-1, in patients with advanced hematologic malignancies," Clinical Cancer Research, vol. 14, no. 10, pp. 3044-3051, 2008.

[54] M. Sznol, J. Powderly, D. Smith et al., "Safety and antitumor activity of biweekly MDX-1106 (Anti-PD-1, BMS936558/ONO-4538) in patients with advanced refractory malignancies," Journal of Clinical Oncology, vol. 28, supplement 15s, abstract 2506, 2010.

[55] M. A. Curran, W. Montalvo, H. Yagita, and J. P. Allison, "PD1 and CTLA-4 combination blockade expands infiltrating T cells and reduces regulatory $\mathrm{T}$ and myeloid cells within B16 melanoma tumors," Proceedings of the National Academy of Sciences of the United States of America, vol. 107, no. 9, pp. 4275-4280, 2010.

[56] S. D. Gray-Owen and R. S. Blumberg, "CEACAM1: contactdependent control of immunity," Nature Reviews Immunology, vol. 6, no. 6, pp. 433-446, 2006.

[57] K. Kuespert, S. Pils, and C. R. Hauck, "CEACAMs: their role in physiology and pathophysiology," Current Opinion in Cell Biology, vol. 18, no. 5, pp. 565-571, 2006.

[58] A. Ebrahimnejad, T. Streichert, P. Nollau et al., "CEACAM1 enhances invasion and migration of melanocytic and melanoma cells," American Journal of Pathology, vol. 165, no. 5, pp. 1781-1787, 2004.

[59] T. Gambichler, S. Grothe, S. Rotterdam, P. Altmeyer, and A. Kreuter, "Protein expression of carcinoembryonic antigen cell adhesion molecules in benign and malignant melanocytic skin lesions," American Journal of Clinical Pathology, vol. 131, no. 6, pp. 782-787, 2009.

[60] A. Thies, I. Moll, J. Berger et al., "CEACAM1 expression in cutaneous malignant melanoma predicts the development of metastatic disease," Journal of Clinical Oncology, vol. 20, no. 10, pp. 2530-2536, 2002.
[61] J. Brümmer, A. Ebrahimnejad, R. Flayeh et al., "cis interaction of the cell adhesion molecule CEACAM1 with itegrin $\beta 3$," American Journal of Pathology, vol. 159, no. 2, pp. 537-546, 2001.

[62] G. Markel, D. Wolf, J. Hanna et al., "Pivotal role of CEACAM1 protein in the inhibition of activated decidual lymphocyte functions," Journal of Clinical Investigation, vol. 110, no. 7, pp. 943-953, 2002.

[63] G. Markel, R. Seidman, Y. Cohen et al., "Dynamic expression of protective CEACAM1 on melanoma cells during specific immune attack," Immunology, vol. 126, no. 2, pp. 186-200, 2009.

[64] G. Markel, R. Seidman, N. Stern et al., "Inhibition of human tumor-infiltrating lymphocyte effector functions by the homophilic carcinoembryonic cell adhesion molecule 1 interactions," Journal of Immunology, vol. 177, no. 9, pp. 6062-6071, 2006.

[65] G. Markel, H. Mussaffi, K. L. Ling et al., "The mechanisms controlling NK cell autoreactivity in TAP2-deficient patients," Blood, vol. 103, no. 5, pp. 1770-1778, 2004.

[66] G. Markel, R. Gruda, H. Achdout et al., "The critical role of residues $43 \mathrm{R}$ and $44 \mathrm{Q}$ of carcinoembryonic antigen cell adhesion molecules- 1 in the protection from killing by human NK cells," Journal of Immunology, vol. 173, no. 6, pp. 37323739, 2004.

[67] N. Azuz-Lieberman, G. Markel, S. Mizrahi et al., "The involvement of NK cells in ankylosing spondylitis," International Immunology, vol. 17, no. 7, pp. 837-845, 2005.

[68] G. Markel, R. Ortenberg, R. Seidman et al., "Systemic dysregulation of CEACAM1 in melanoma patients," Cancer Immunology, Immunotherapy, vol. 59, no. 2, pp. 215-230, 2010.

[69] G. Markel, N. Lieberman, G. Katz et al., "CD66a interactions between human melanoma and NK cells: a novel class I MHCindependent inhibitory mechanism of cytotoxicity," Journal of Immunology, vol. 168, no. 6, pp. 2803-2810, 2002.

[70] G. Markel, H. Achdout, G. Katz et al., "Biological function of the soluble CEACAM1 protein and implications in TAP2deficient patients," European Journal of Immunology, vol. 34, no. 8, pp. 2138-2148, 2004.

[71] Z. Chen, L. Chen, S. W. Qiao, T. Nagaishi, and R. S. Blumberg, "Carcinoembryonic antigen-related cell adhesion molecule 1 inhibits proximal TCR signaling by targeting ZAP-70," Journal of Immunology, vol. 180, no. 9, pp. 6085-6093, 2008.

[72] T. Nagaishi, L. Pao, S. H. Lin et al., "SHP1 phosphatasedependent $\mathrm{T}$ cell inhibition by CEACAM1 adhesion molecule isoforms," Immunity, vol. 25, no. 5, pp. 769-781, 2006.

[73] N. Stern, G. Markel, T. I. Arnon et al., "Carcinoembryonic antigen (CEA) inhibits NK killing via interaction with CEArelated cell adhesion molecule 1," Journal of Immunology, vol. 174, no. 11, pp. 6692-6701, 2005.

[74] M. J. Moller, R. Kammerer, F. Grunert, and S. von Kleist, "Biliary glycoprotein (BGP) expression on T-cells and on a natural-killer-cell sub-population," International Journal of Cancer, vol. 65, no. 6, pp. 740-745, 1996.

[75] R. Ortenberg, Y. Sapir, L. Raz, L. Hershkovitz, A. B. Arav et al., "Novel immunutherapy for malignant melanoma with a monoclonal antibody that blocks CEACAM1 homophilic interactions," Molecular Cancer Therapeutics. In press.

[76] M. E. Dudley, J. R. Wunderlich, J. C. Yang et al., "Adoptive cell transfer therapy following non-myeloablative but lymphodepleting chemotherapy for the treatment of patients with refractory metastatic melanoma," Journal of Clinical Oncology, vol. 23, no. 10, pp. 2346-2357, 2005. 
[77] M. E. Dudley, J. C. Yang, R. Sherry et al., "Adoptive cell therapy for patients with metastatic melanoma: evaluation of intensive myeloablative chemoradiation preparative regimens," Journal of Clinical Oncology, vol. 26, no. 32, pp. 5233-5239, 2008.

[78] M. J. Besser, R. Shapira-Frommer, A. J. Treves et al., "Clinical responses in a phase II study using adoptive transfer of shortterm cultured tumor infiltration lymphocytes in metastatic melanoma patients," Clinical Cancer Research, vol. 16, no. 9, pp. 2646-2655, 2010.

[79] D. J. Schwartzentruber, S. S. Hom, R. Dadmarz et al., "In vitro predictors of therapeutic response in melanoma patients receiving tumor-infiltrating lymphocytes and interleukin-2," Journal of Clinical Oncology, vol. 12, no. 7, pp. 1475-1483, 1994.

[80] R. A. Morgan, M. E. Dudley, J. R. Wunderlich et al., "Cancer regression in patients after transfer of genetically engineered lymphocytes," Science, vol. 314, no. 5796, pp. 126-129, 2006.

[81] L. A. Johnson, R. A. Morgan, M. E. Dudley et al., "Gene therapy with human and mouse T-cell receptors mediates cancer regression and targets normal tissues expressing cognate antigen," Blood, vol. 114, no. 3, pp. 535-546, 2009.

[82] C. A. Ramos and G. Dotti, "Chimeric antigen receptor (CAR)engineered lymphocytes for cancer therapy," Expert Opinion on Biological Therapy, vol. 11, no. 7, pp. 855-873, 2011.

[83] E. Yvon, M. del Vecchio, B. Savoldo et al., "Immunotherapy of metastatic melanoma using genetically engineered GD2specific T cells," Clinical Cancer Research, vol. 15, no. 18, pp. 5852-5860, 2009.

[84] W. Peng, Y. Ye, B. A. Rabinovich et al., "Transduction of tumor-specific $\mathrm{T}$ cells with CXCR2 chemokine receptor improves migration to tumor and antitumor immune responses," Clinical Cancer Research, vol. 16, no. 22, pp. 5458$5468,2010$.

[85] C. Zito and H. M. Kluger, "Immunotherapy for metastatic melanoma," Journal of Cellular Biochemistry, vol. 113, no. 3, pp. 725-734, 2012.

[86] A. Hoos, A. M. Eggermont, S. Janetzki et al., "Improved endpoints for cancer immunotherapy trials," Journal of the National Cancer Institute, vol. 102, no. 18, pp. 1388-1397, 2010.

[87] P. Kantoff, C. Higano, N. Shore et al., "Sipuleucel-T immunotherapy for castration-resistant prostate cancer," The New England Journal of Medicine, vol. 363, no. 5, pp. 411-422, 2010.

[88] S. L. Topalian, C. G. Drake, and D. M. Pardoll, "Targeting the PD-1/B7-H1(PD-L1) pathway to activate anti-tumor immunity," Current Opinion in Immunology, vol. 24, no. 2, pp. 207212, 2012.

[89] Toxicity and Activity of anti-PD-1 in Phase 1 Clinical Trials, in Proceedings of the 102nd Annual Meeting of American Association for Cancer Research (AACR '11), Orlando, Fla, USA, April 2011. 


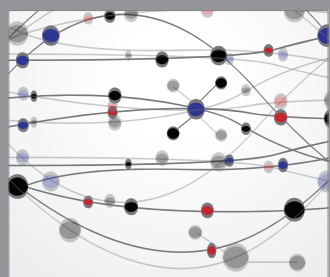

The Scientific World Journal
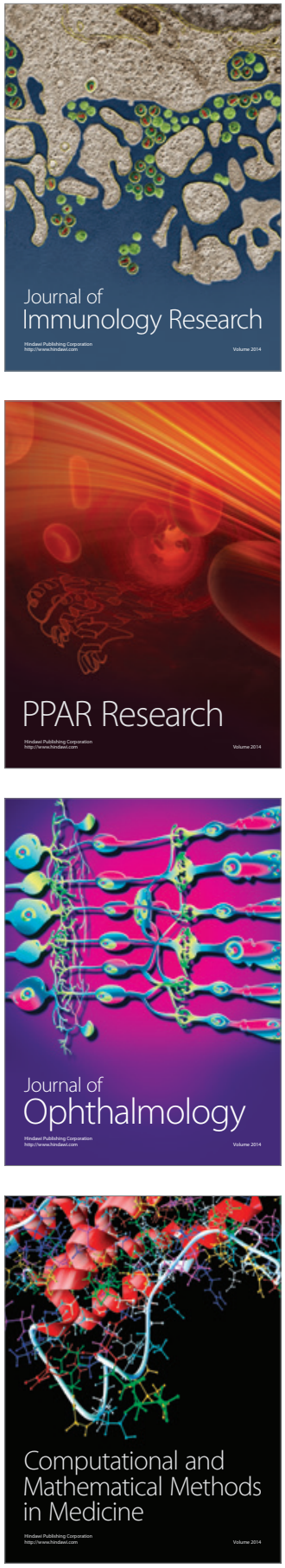

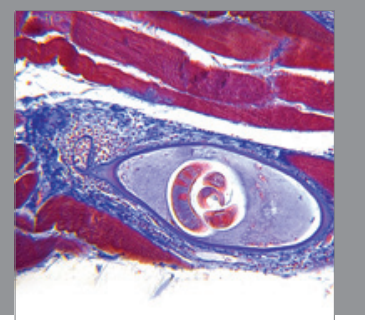

Gastroenterology

Research and Practice
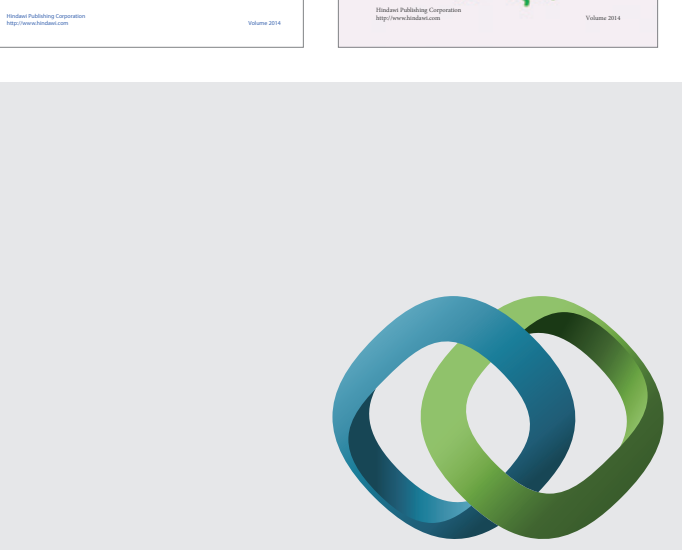

\section{Hindawi}

Submit your manuscripts at

http://www.hindawi.com
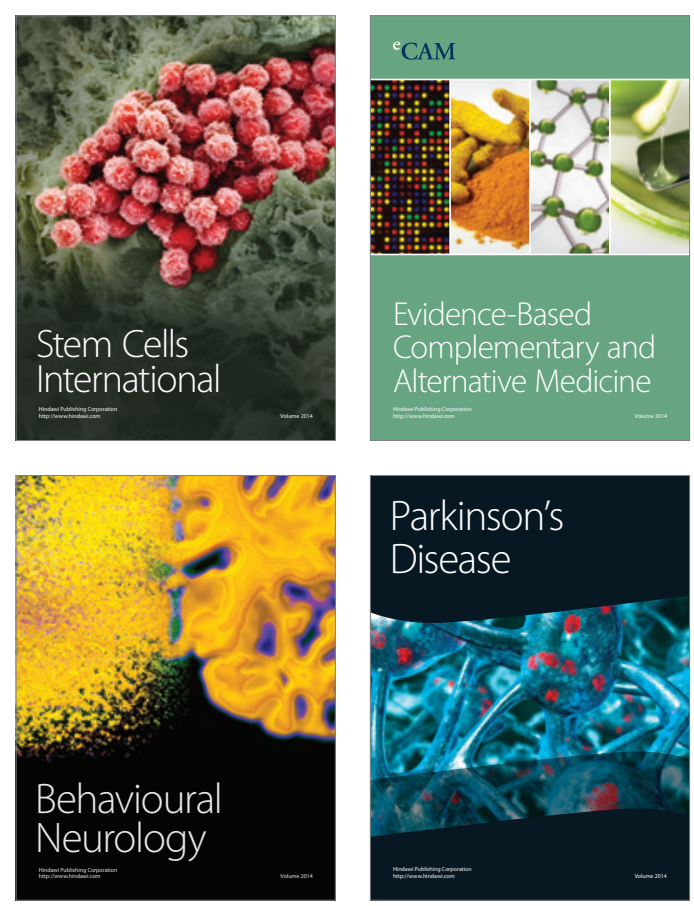

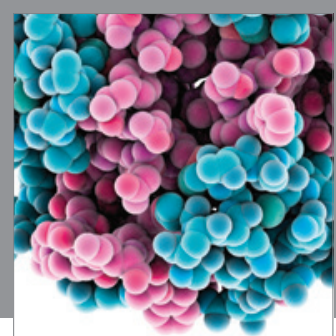

Journal of
Diabetes Research

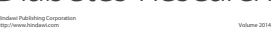

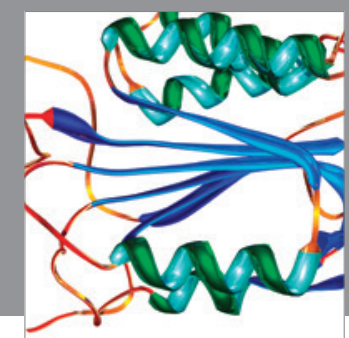

Disease Markers
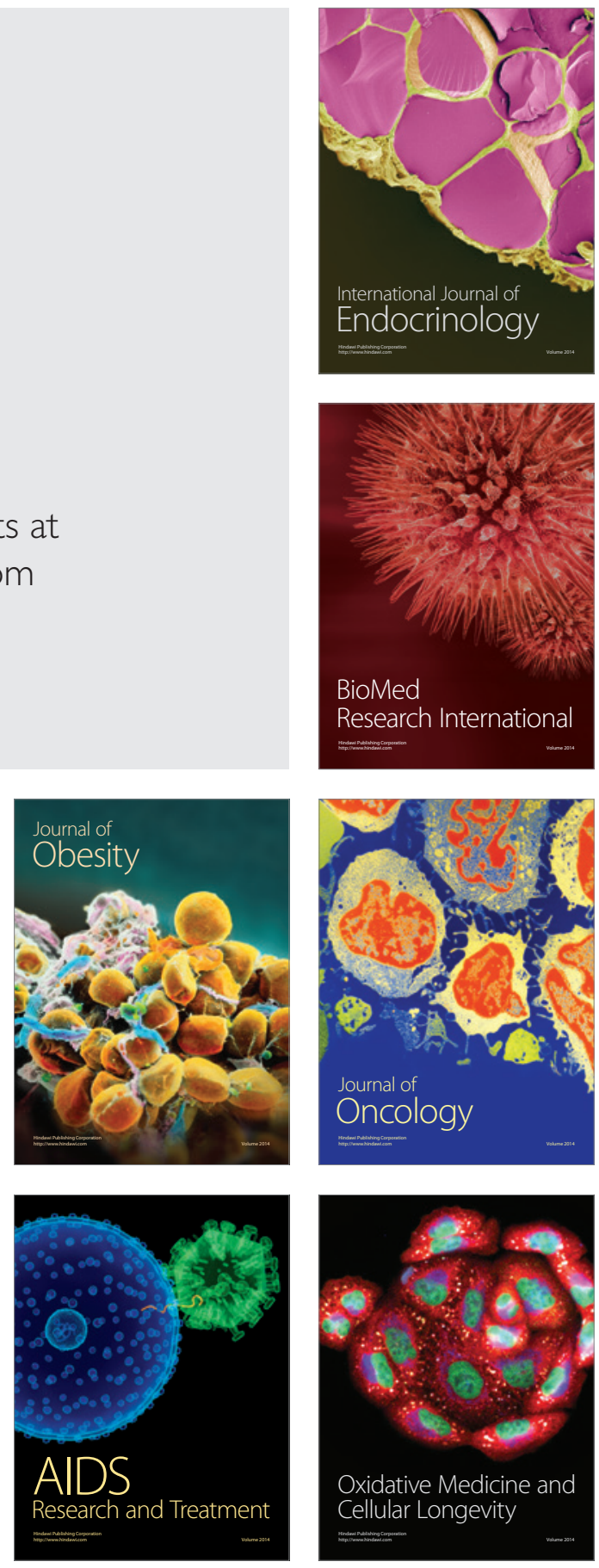